Edilson da Silva Brabo 1 Elisabeth de Oliveira Santos 1 Iracina Maura de Jesus 1 Artur Fernando Mascarenhas 2 Kleber Freitas Faial 1

\section{Níveis de mercúrio em peixes consumidos pela comunidade indígena de Sai Cinza na Reserva Munduruku, Município de J acareacanga, Estado do Pará, Brasil}

\author{
Mercury levels in fish consumed by the Sai Cinza \\ indigenous community, Munduruku Reservation, \\ J acareacanga County, State of Pará, Brazil
}

1 Instituto Evandro Chagas, Coordenação de Ecologia Humana e Meio Ambiente, Fundação Nacional de Saúde. Av. Almirante Barroso 492, Marco, Belém, PA 66090-000, Brasil. 2 Secretaria de Estado de Indústria, Comércio e Mineração.

Av. Presidente Vargas 1020 Centro, Belém, PA 66017-000, Brasil.

\begin{abstract}
This study evaluated fish consumption and mercury levels in fish consumed by an indigenous community in the State of Pará. Eighty fish samples were collected (barbado, surubim, traíra, tucunaré, piranha, aruanã, caratinga, aracu, mandiá, jandiá, and pacu). Mercury analysis was performed using a Mercury Analyzer HG-3500. Average mercury concentration in carnivorous species was $0.293 \mu \mathrm{g} / \mathrm{g}$ (SD $=0.104$ ), while in non-carni vorous species it was $0.112 \mu \mathrm{g} / \mathrm{g}$ ( $S D=0.036)$. Brazilian legislation establishes a maximum permissible limit of $0.5 \mu \mathrm{g} / \mathrm{g}$ for fish consumption. No significant correlation was found between fish length or weight and mercury concentration. Types of fish most frequently consumed by the community were tucunaré, pacu, jaraqui, traíra, aracu, matrinchã, and caratinga. Carni vorous species, especially tucunaré and traíra, amongst the most frequently eaten, had higher mercury levels than non-carnivorous species. Frequency of consumption is crucial to assess the risk of mercury contamination in communities who lack alternative food sources.

Key words Mercury; South American Indians; Fish

Resumo O estudo consiste em avaliar os níveis de mercúrio no pescado consumido pela comunidade indígena de Sai Cinza (Reserva Munduruku) no Estado do Pará, e associá-los com os hábitos de consumo da população. Um total de oitenta espéci mes de pei xes foram capturados. As determinações de $\mathrm{Hg}$ foram realizadas por absorção atômica. A concentração média de Hg nas espécies carnívoras foi de $0,293 \mu \mathrm{g} / \mathrm{g}(\mathrm{DP}=0,104)$ enquanto nas não carnívoras foi de $0,112 \mu \mathrm{g} / \mathrm{g}$ $(D P=0,036)$. As espécies referidas como de mai or consumo ente os 330 indi víduos entrevistados foram: tucunaré, pacu, jaraqui, traíra, aracu, matrinchã e caratinga. As espécies com concentrações mais el evadas de Hg foram tucunaré e traíra, que estão entre os peixes mais consumidos. A freqüência de consumo constitui-se num fator importante na avaliação de risco de contaminação por mercúrio em comuni dades que não têm outras alternati vas de alimentação.

Palavras-chave Mercúrio; Índios Sul-Americanos; Peixes
\end{abstract}




\section{Introdução}

A participação da atividade garimpeira de ouro na economia da região do Tapajós teve início no final da década de 50, quando foram descobertas as primeiras ocorrências do metal no rio das Tropas, afluente da margem direita do rio Tapajós. Desde então, o garimpo revelou-se como um assunto polêmico, em função da diversidade das questões sociais, políticas, econômicas e dos problemas ambientais gerados, com a utilização do mercúrio $(\mathrm{Hg})$ no processo de extração e a emissão do mercúrio para o ambiente (Rodrigues et al., 1994).

Uma das vias de liberação de $\mathrm{Hg}$ para o ambiente ocorre na etapa de concentração dos sedimentos aluvionares, coluvionares e do fundo dos rios. Nessa fase, o Hg é adicionado aos sedimentos e podem ocorrer perdas de Hg para os rios e solos. Outra forma de emissão de $\mathrm{Hg}$ acontece durante a queima do amálgama (liga metálica de ouro-mercúrio) realizada ao ar livre. Neste processo, os vapores de $\mathrm{Hg}$ são Iançados na atmosfera e posteriormente são precipitados com as chuvas contaminando rios, solos e vegetação (Lacerda, 1990).

Quando o ouro é comercializado nas casas de compra e venda, centros urbanos ou nas "currutelas" (pequenos núcleos urbanos de apoio aos garimpos), há a necessidade da "requeima". Durante este procedimento, ocorre a contaminação atmosférica desses ambientes fechados que, na maioria das vezes, não possuem sistemas de exaustão adequados. Nessas condições ocorre a exposição ao vapor de Hg dos indivíduos que trabal ham nestes locais (Silva, 1993).

As informações sobre a quantidade de mercúrio lançado para o ambiente aquático e atmosférico, sobretudo na década de 80 , são contraditórias. Essas estimativas tomam como base a produção de ouro, considerando-se que, em média, para cada kg de ouro extraído a mesma quantidade de mercúrio é utilizada. Nesse período, as estimativas oficiais são da ordem de 700 toneladas de ouro produzidas na Amazônia (DNPM , 1990). Os dados não oficiais apontam uma produção de ouro que variou entre $1000 \mathrm{e}$ 2000 toneladas.

As características da região amazônica, associadas ao conhecimento ainda incompleto do ciclo do mercúrio em florestas tropicais úmidas, mostram a complexidade dos estudos sobre contaminação mercurial e a dificuldade da avaliação dos riscos da exposição dos diferentes grupos populacionais na região (Hacon, 1997; Santos, 1993).

O risco de contaminação por mercúrio, originada nos garimpos de ouro, tem sido alvo de preocupação na área ambiental e da saúde pública. Em relação às populações humanas, uma das possíveis vias de exposição é por meio da ingestão de alimentos contaminados. Este aspecto cresce em importância quando se trata de comunidades ribeirinhas, especialmente indígenas, onde o pescado é a base da dieta protéica diária (Gonçalves, 1993; Bidone et al., 1995; Bidone et al., 1997; Barbosa et al., 1997; Boischio \& Cernichiari, 1998).

Considerando os hábitos alimentares das comunidades indígenas da região, as informações sobre os níveis de mercúrio no pescado e outros organismos aquáticos utilizados na alimentação são de extrema importância na avaliação do risco a que estes indivíduos estão submetidos (Hacon et al., 1997).

Os dados disponíveis, até o momento, ainda não são suficientes para avaliar com exatidão o grau de comprometimento da biota, sobretudo peixes, de áreas direta ou indiretamente afetadas pela atividade garimpeira na região amazônica (Aula et al., 1994; Malm et al., 1995; Lebel et al., 1997; Brabo et al., 1998; Kehrig et al., 1998; Mascarenhas et al., 1998).

Vários fatores influenciam o grau de exposição dos indivíduos, dentre eles merecem destaque a freqüência da ingestão de pescado; preferência por determinadas espécies; tamanho dos espécimes e o nível trófico (Phillips et al., 1980; Paradis et al., 1997).

Na região amazônica foram desenvolvidos poucos estudos envolvendo a especiação química do mercúrio, sobretudo em organismos aquáticos. No entanto, as pesqui sas realizadas em peixes têm demonstrado que cerca de 60 a $95 \%$ do mercúrio total, em tecido muscular, ocorrem na forma de metil mercúrio, que éuma das formas mais tóxicas para o homem (Westoo, 1966; Cappon \& Smith, 1981; WHO, 1990; Akagi et al., 1996).

Este estudo é parte integrante da investigação de saúde ambiental que o Instituto Evandro Chagas, por intermédio da Coordenação de Ecologia Humana e Meio Ambiente (COEH$\mathrm{MA}$ ), vem desenvolvendo em conjunto com outras instituições, sobre as condições de vida e saúde de diferentes tipos de populações submetidas à influência da atividade garimpeira, na bacia hidrográfica do rio Tapajós, Estado do Pará.

Nesse trabalho, são apresentadas as concentrações de mercúrio nas diferentes espécies de peixes capturadas nos principais locais de pesca da comunidade do posto indígena Sai Cinza, associadas à preferência de consumo de peixes pela população. 
Área de estudo

A investigação foi realizada na área do Posto Indígena Sai Cinza, Reserva Munduruku, situada no sudoeste do Estado do Pará, no Município de Jacareacanga, margem esquerda do rio Tapajós entre os paralelos 6014'06"S e 57०46' 31"W, em área de risco de contaminação pelo mercúrio utilizado na garimpagem (Figura 1).

O Posto Indígena Sai Cinza está situado a cerca de $15 \mathrm{~km}$ a montante da sede do Município de Jacareacanga. O aldeamento abriga 63 edificações distribuídas ao longo das margens do rio Tapajós que ocupa um espaço de 1205 metros. A população residente na aldeia é de 560 habitantes e tem como atividade principal a pesca de subsistência. Há presença de garimpos na área da reserva, porém o envolvimento dos indígenas do Posto Sai Cinza nesta atividade é restrito.

\section{Materiais e métodos}

A coleta de peixes foi realizada nos lagos situados nas proximidades do Posto Indígena Sai Cinza. Estes, por estarem situados em áreas inundáveis, durante o período das cheias se integram ao leito natural das drenagens maiores, e nos meses de menor precipitação pluviométrica ficam apenas interligados a essas drenagens maiores por pequenos canais.

Um total de 80 de peixes de diferentes espécies e hábitos alimentares foram analisados. As espécies carnívoras estão representadas pelo tucunaré, traíra, barbado, piranha, surubim e aruanã. Os peixes de hábitos não carnívoros incluem os detritívoros: caratinga; herbívoros: aracu, pacu e mandiá; e onívoros: jandiá (Tabela 1).

Logo após a coleta dos espécimes, foi preenchida uma ficha contendo informações sobre identificação, dimensões, peso, modo de conservação, procedência, entre outras. Em seguida, foi retirada uma quantidade do tecido muscular próximo à espinha dorsal que variou entre 50 a 100 gramas, a qual foi mantida congelada até ser transportada em ambiente com gelo para o laboratório em Belém. As amostras foram mantidas a $-20 \circ \mathrm{C}$ até a execução dos procedimentos analíticos.

Foi realizado um inquérito epidemiológico na população com aplicação individual de um questionário a 330 pessoas residentes, o que representa cerca de $60 \%$ da população do Posto Indígena Sai Cinza. As questões abrangidas eram do tipo fechadas e incluíam dados sobre identificação, hábitos alimentares e morbida- de. Neste trabalho enfocam-se somente as informações referentes aos hábitos alimentares quanto às espécies de peixes mais consumidas e a freqüência de consumo.

Os procedimentos analíticos para quantificação de Hg total foram os preconizados por Akagi et al. (1995). As determinações de Hg foram realizadas por espectrometria de absorção atômica sem chama, utilizando-se o modelo Mercury Analizer Hg 3500, cujo princípio de funcionamento é mostrado na Figura 2.

Exercícios de intercalibração são realizados com o National Institute for Minamata Disease - NIMD (Kumamoto, Japão), Centro de Estudos e Análises Mercuriais da Amazônia CEAMA (Departamento Nacional da Produção Mineral/PA), Universidade Federal do Pará (UFPa) e a Fundação Universidade Federal de Rondônia (UNIR).

\section{Resultados}

Na Tabela 1 são apresentados os nomes popular e científico dos espécimes analisados, assim como as concentrações médias e variações de mercúrio.

Os níveis de mercúrio nos peixes coletados estão relacionados aos hábitos alimentares das espécies. A concentração média de mercúrio nos peixes carnívoros foi de 0,293 $\mu \mathrm{g} / \mathrm{g}$ (DP = $0,104)$. Deste grupo, o barbado, o surubim e a traíra apresentaram concentrações médias no intervalo de 0,322 a 0,419 $\mu \mathrm{gHg} / \mathrm{g}$. Os dados analíticos indicaram que apenas um espécime de traíra, com 0,546 $\mu \mathrm{gHg} / \mathrm{g}$, ultrapassou o valor de referência $(0,5 \mu \mathrm{gHg} / \mathrm{g}$ com consumo semanal de 400 gramas) adotado como limite máximo permitido pelo Ministério da Saúde, para ingestão de pescado (Brasil, 1975). Nas amostras de espécies não carnívoras a média foi de $0,112 \mu \mathrm{gHg} / \mathrm{g}$ (DP =0,036). Neste grupo nenhuma das amostras atingiu o valor de referência acima citado.

O resultado da análise de correlação entre a concentração de mercúrio, o tamanho e o peso das espécies estudadas não mostrou significância estatística para o grupo dos peixes não carnívoros (tamanho: $r=0,0780$; peso: $r=-0,0928$ ). Para ambas as variáveis, o coeficiente encontrado mostrou-se abaixo do valor crítico ( $r=0,2875)$ para o nível de significância utilizado ( $g l=47 ; p<0,05$ )

Nesse grupo, a espécie caratinga apresentou correlação positiva moderada entre os níveis de mercúrio e as mesmas variáveis citadas (tamanho: $r=0,6620$; peso: $r=0,5440$ ), com coeficientes situados acima do valor crí- 
Figura 1

Mapa de localização da Área Indígena Sai Cinza.
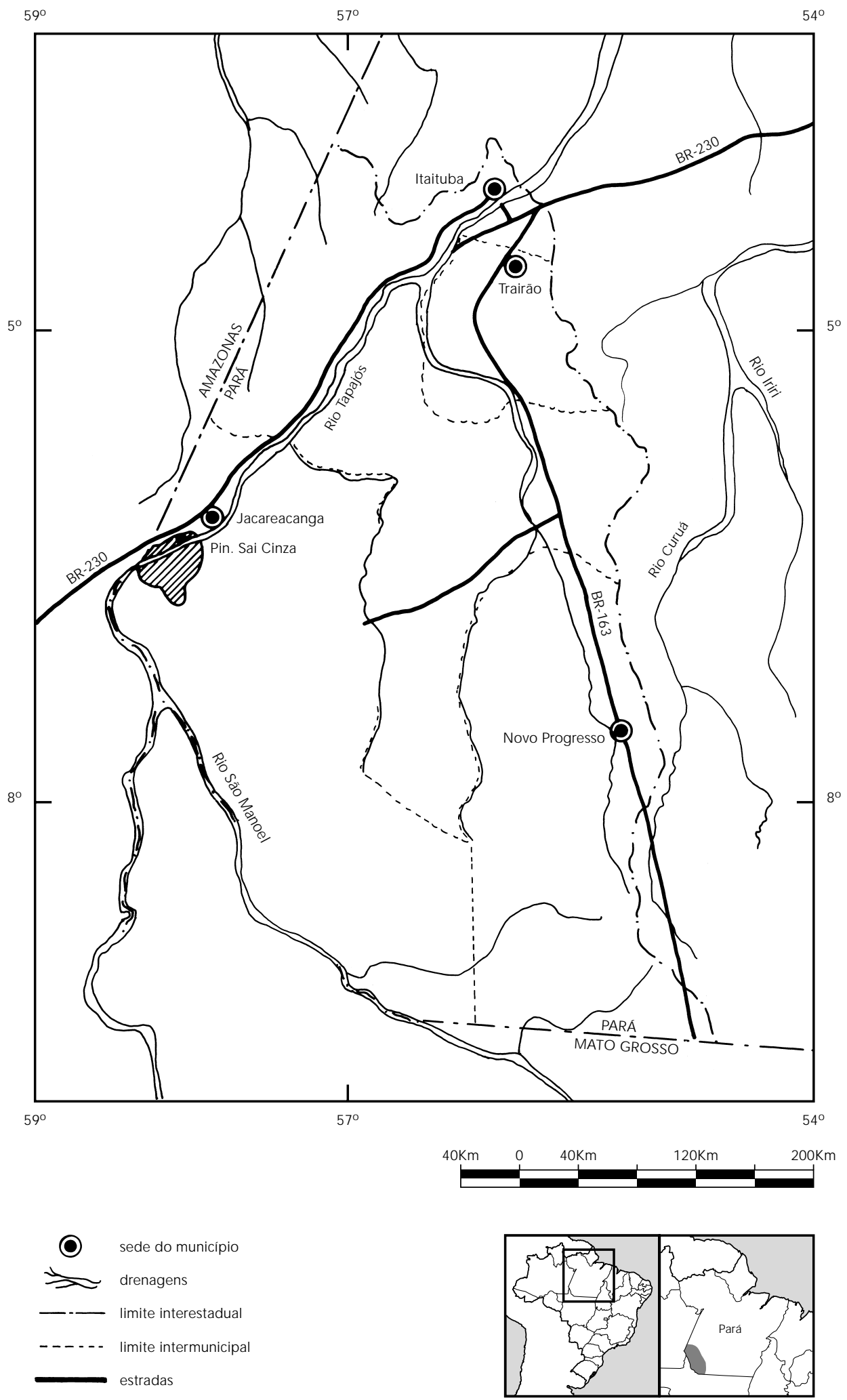

área indígena Sai Cinza 
Tabela 1

Concentrações de $\mathrm{Hg}$ nas diferentes espécies de peixes do Posto Indígena Sai Cinza, Reserva Munduruku (V acareacanga - Pará), 1995.

\begin{tabular}{lllrl}
\hline Nome Popular & Nome Científico & Espécimes $(\mathrm{n})$ & \multicolumn{2}{c}{$\begin{array}{c}\text { Concentração de Hg }(\mathrm{g} / \mathrm{g}) \\
\text { Faixa }\end{array}$} \\
& & & $0,349-0,474$ & $0,419 \pm 0,064$ \\
\hline Barbado (C) & Pinirampus pirinampu & 03 & 0 & $0,385 \pm 0,000$ \\
Surubim (C) & Pseudoplatystoma fasciatum & 01 & $0,160-0,546$ & $0,322 \pm 0,133$ \\
Traíra (C) & Hoplias malabaricus & 07 & $0,173-0,338$ & $0,267 \pm 0,049$ \\
Tucunaré (C) & Cichla ocellaris & 17 & $0,065-0,450$ & $0,219 \pm 0,204$ \\
Piranha (C) & Serrasalmus sp. & 03 & 0 & $0,174 \pm 0,000$ \\
Aruanã (C) & Osteoglossum bicirrhosum & 01 & $0,067-0,198$ & $0,120 \pm 0,039$ \\
Caratinga (NC) & Satanoperca sp. & 18 & $0,074-0,201$ & $0,112 \pm 0,028$ \\
Jaraqui (NC) & Semaprochilodus brama & 22 & $0,097-0,120$ & $0,108 \pm 0,016$ \\
Aracu (NC) & Leporinus sp. & 02 & 0 & $0,095 \pm 0,000$ \\
Mandiá (NC) & Pimelodus omatus & 01 & $0,051-0,130$ & $0,093 \pm 0,040$ \\
Jandiá (NC) & Leiaurius marmoratus & 03 & $0,026-0,057$ & $0,042 \pm 0,022$ \\
Pacu (NC) & Mylossoma sp. & 02 & & \\
Total & & 80 & &
\end{tabular}

C = espécies carnívoras; N C = espécies "não carnívoras".

Figura 2

Diagrama esquemático do princípio de funcionamento do Mercury Analizer $\mathrm{Hg} 3500$.

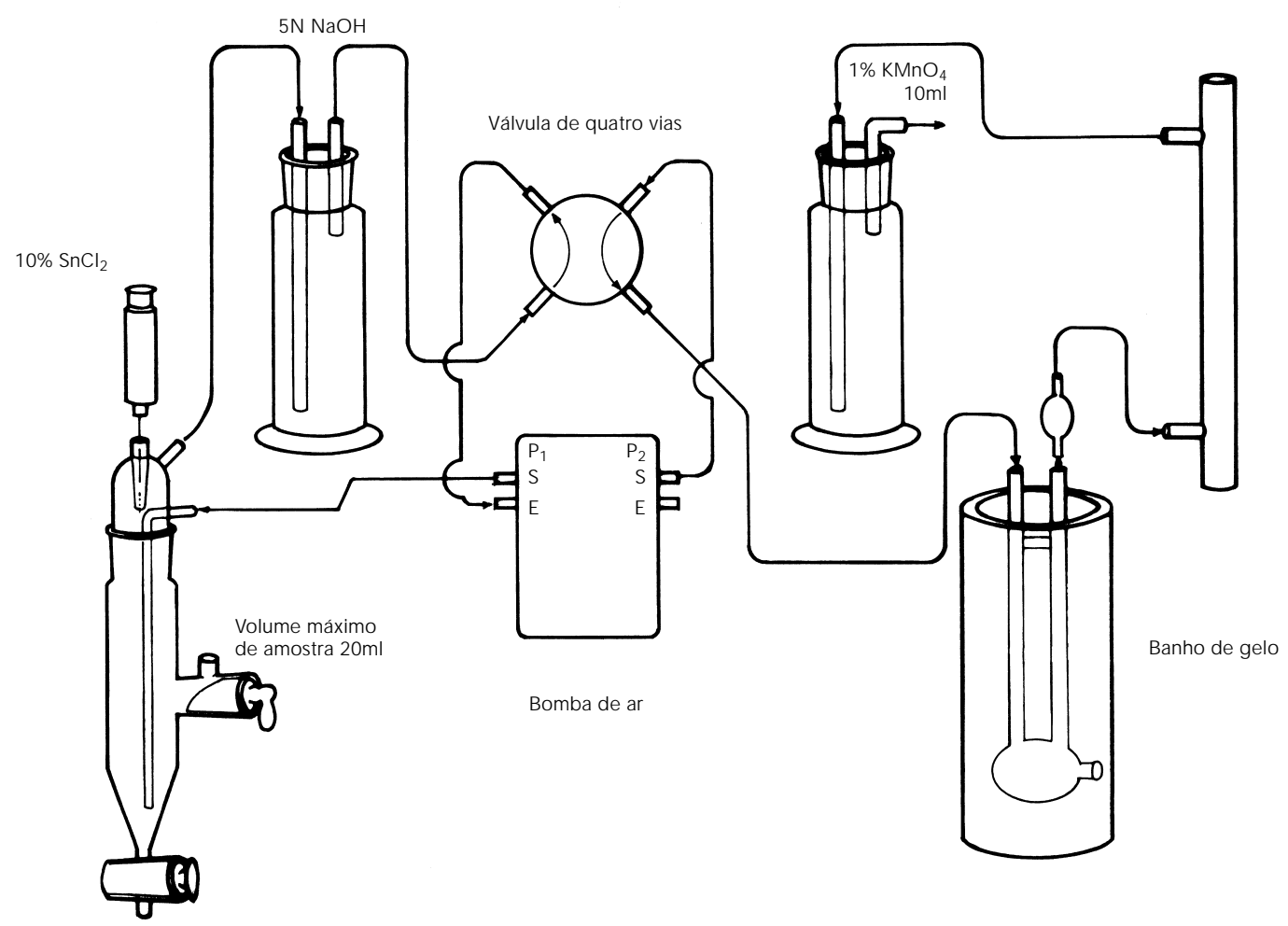

E: entrada; S: saída. 
tico ( $r=0,4683 ; \mathrm{gl}=16 ; \mathrm{p}<0,05)$. No grupo dos peixes carnívoros a correlação observada entre a concentração de mercúrio e o tamanho e peso das espécies avaliadas mostrou-se positiva e moderada (tamanho: $r=0,6303$; peso: $r=0,5182$ ), sendo os coeficientes superiores ao valor considerado crítico para o grau de liberdade utilizado ( $g l=29 ; r=0,3809 ; p \varangle 0,05$ ). Entre os representantes deste grupo, destaca-se a correlação dos níveis de mercúrio observados na espécie traíra em relação ao tamanho e peso dos espécimes (tamanho: $r=0,7279$; peso: $r=0,6590$ ). Porém, em ambos os casos, o coeficiente calculado mostrou-se abaixo do valor crítico estabelecido para o grau de liberdade verificado ( $\mathrm{gl}=5 ; \mathrm{r}=0,7545 ; \mathrm{p} \varangle 0,05$ ).

Este estudo demonstrou que o pescado representa a dieta básica diária de $100 \%$ dos indivíduos entrevistados (330/560), sendo consumido no mínimo três vezes ao dia. Os peixes referidos como de maior consumo foram: tucunaré $86,4 \%$ (285/330), pacu 82,1\% (271/330), jaraqui 79,7\% (263/330), traíra 65,5\% (216/ $330)$, aracu 54,2\% (179/330), matrinchã 15,8\% (52/330) e caratinga 4,2\% (14/330).

\section{Discussão e conclusão}

As concentrações mais elevadas de $\mathrm{Hg}$ foram verificadas nas espécies tucunaré e traíra (carnívoras), as quais estão entre as mais consumidas pela população. Dentre as não carnívoras analisadas, cujas concentrações foram relativamente mais baixas, sobressaem-se as espécies jaraqui e aracu, também de elevado consumo.

Assumindo-se que 60 a 95\% do mercúrio encontrado nos tecidos de peixes estão na forma metilada, os níveis de Hg nas espécies tendem a aumentar devido aos processos de organificação em ambientes aquáticos no transporte do metal para as redes tróficas, a facilidade de absorção das espécies organomercuriais e a

\section{Agradecimentos}

Os autores agradecem o apoio financeiro do Instituto Evandro Chagas e da União Européia. baixa taxa de excreção, associados aos processos de bioacumulação e disponibilidade do metal no meio aquático.

Informações sobre o peso, o tamanho e os hábitos alimentares das espécies, são importantes na avaliação dos processos de bioacumulação de Hg, embora neste estudo não se tenha verificado, de modo geral, a predominância de correlação forte entre essas variáveis e os níveis de mercúrio em tecido muscular nas espécies avaliadas.

A mesma observação, entretanto, foi feita em outros estudos principalmente nas bacias dos rios Madeira e Tapajós, o que pode estar associado não só à variabilidade na dieta alimentar de cada espécie de peixe e capacidade de migração, mas também, a outras variáveis do meio aquático, tais como: dinâmica de fluxo, espessura da lâmina d'água, níveis de mercúrio nos sedimentos de fundo e em suspensão e nas comunidades planctônicas e bentônicas (Lacerda et al., 1988; Reuther, 1994).

Ainda que o pescado consumido pela população de Sai Cinza esteja com concentrações médias de mercúrio abaixo do que estabelece a legislação, a freqüência de consumo e a quantidade diária ingerida é um componente essencial na avaliação do risco de contaminação por mercúrio.

Desta forma, a exposição contínua ao mercúrio pela via alimentar, mesmo consi derando as baixas concentrações do metal encontradas nos peixes, permitem a hipótese de que no decorrer do tempo, esta situação se agrave, principalmente em comunidades onde além do pescado, não é comum uma dieta diversificada.

A intensificação dos estudos na região amazônica, mediante coletas sistemáticas e de maior abrangência, poderiam contribuir para o estabelecimento de programas de orientação referentes aos hábitos de consumo de peixes por comunidades ribeirinhas, expostas aos riscos da garimpagem.
Referências

AKAGI, H.; MALM, O.; KINJO, Y.; HARADA, M.; BRANCHES, F. J. P.; PFEIFFER, W. C. \& KATO, H., 1995. Methylmercury pollution in the Amazon, Brazil. Science of the Total Environment, 175:85-95.

AKAGI, H.; MALM , O. \& BRANCHES, F. J. P., 1996. Human Exposure to mercury due to mining in the Amazon, Brazil - A review. Environmental Sciences, 3:199-211.

AULA, I.; BRAUNSCHWEILER, H.; LEINO, T.; MALIN, I.; PORVARI, P.; HATANAKA, T.; LODENIUS, M. \& JURAS, A., 1994. Levels of mercury in the Tucuruí 
reservoir and its surrounding area in Pará, Brazil. In: Mercury Pollution Integration and Synthesis (C. J. Watras \& J. W. Huckabee, eds.), pp. 21-40, Monterey: CRC Press.

BARBOSA, A. C.; GARCIA, A. M. \& SOUZA, J. R., 1997. Mercury contamination in hair of riverine of Apiacás Reserve in the Brazilian Amazon. Water, Air and Soil Pollution, 97:1-8.

BIDONE, E. D.; SOUZA, T. M. C.; MASCARENHAS, A. F. S. \& RODRIGUES, R. M., 1995. Monitoramento deÁguas e Peixes na Bacia do Tapajós. Belém: Secretaria de Estado de Indústria, Comércio e Mineração - Seicom/Governo do Estado do Pará.

BIDONE, E. D.; CASTILHOS, Z. C.; SANTOS, T. J. S.; SOUZA, T. M. C. \& LACERDA, L. D., 1997. Fish contamination and human exposure to mercury in Tartarugalzinho River, Amapá State, Northern Amazon, Brazil. A screening approach. Water, Air and Soil Pollution, 97:9-15.

BOISCHIO, A. A. P. \& CERNICHIARI, E., 1998. Longitudinal hair mercury concentration in riverside mothers along the upper Madeira river (Brazil). Environmental Research, 77:79-83.

BRABO, E.; SANTOS, E. C.; JESUS, I. M .; MASCARENHAS, A. F. S\& FAIAL, K. F., 1998. Verificação dos níveis de mercúrio no pescado consumido pela comunidade ribeirinha de Santana de Ituqui Bacia do rio Amazonas - Santarém, PA: resultados preliminares. Revista da Sociedade Brasileira de Medicina Tropical, 31(Sup. 1):40.

BRASIL, 1975. Ministério da Saúde, Resolução no 18/ 75 da Comissão Nacional de Normas e Padrões para Alimentos. Diário Oficial da União. Brasília, pp. 16.378, 09 dez. 1975. Seção 1.

CAPPON, C. J. \& SMITH, C., 1981. Mercury and selenium content and chemical form in fish muscle. Archives of Environmental Contamination and Toxicology, 10:305-319.

DNPM (Departamento Nacional de Produção Mineral), 1990. Sumário Mineral. Vol. 10. Brasília: Departamento Nacional de Produção Mineral.

GONÇALVES, A., 1993. Saúde, garimpagem e mercúrio entre os Kaiapó-Gorotire. In: Conseqüências da Garimpagem no Âmbito Social eAmbiental da Amazônia (A. Mathis \& R. Rehaag, org.), pp. 6163, Belém: Editora Cejup.

HACON, S., 1997. Avaliação do Risco Potencial para a Saúde Humana na Exposi ção ao Mercúrio na Área Urbana de Alta Floresta. MT - Bacia AmazônicaBrasil. Tese de Doutorado, Niterói: Programa de Pós-graduação em Geoquímica, Universidade Federal Fluminense.

HACON, S.; ROCHEDO, E. R.; CAM POS, R.; ROSALES. G. \& LACERDA, L. D., 1997. Risk assessment of mercury in Alta Floresta. Amazon Basin - Brazil. Water, Air and Soil Pollution, 97:91-105.

KEHRIG, H. A.; MALM, O.; AKAGI, A.; GUIMARÃES, J. R. D. \& TORRES, J. P. M., 1998. Methylmercury in fish and hair samples from the Balbina Reservoir, Brazilian Amazon. Environmental Research, 77: 84-90.

LACERDA, L. D.; PFEIFFER, W. C.; OTT, A. T. \& SI LVEIRA, E. G., 1988. Mercury contamination in the Madeira river, Amazon - Hg imputs to the environmental. Biotropica, 21:91-93.
LACERDA, L. D., 1990. Distribuição e comportamento do mercúrio no ambiente Amazônico. In: Riscose Conseqüências do Uso do Mercúrio (S. Hacon; L. D. Lacerda; W. C. Pfeiffer \& D. Carvalho, org.), pp. 80-90, Brasília: Finep/Ibama/MS/CNPq.

LEBEL, J.; ROULET, M.; MERGLER, D.; LUCOTTE, M. \& LARRIBE, F., 1997. Fish diet and mercury exposure in a riparian amazonian population. Water, Air and Soil Pollution, 97:31-44.

MALM , O.; BRANCHES, F. J. P.; AKAGI, H.; CASTRO, M. B.; PFEIFFER, W. C.; HARADA, M.; BASTOS, W. R. \& KATO, H., 1995. Mercury and Methylmercury in fish and human hair from the Tapajos river basin, Brazil. Science of the Total Environment, 175:141-150.

MASCARENHAS, A. F. S.; BRABO, E.; SANTOS, E. C.; JESUS, I. M. \& FAIAL, K. F., 1998. Níveis de mercúrio em peixes na bacia hidrográfica do Tapajós, Jacareacanga - Itaituba, Pará: resultados preliminares. Revista da Sociedade Brasileira de Medicina Tropical, 31(Sup. 1):41.

PARADIS, S.; WHEATLEY, B.; BOSWELL-PURDY, J.; BÉLISLE, D.; COLE, M.; LICKERS, H.; HAYTON, A. \& DAVIES, K., 1997. Mercury contamination through fish consumption: A model for predicting and preventing hazardous behaviour on a community level. Water, Air and Soil Pollution, 97:147-158.

PHILLIPS, G. R.; LENHART, T. E. \& GREGORY, R. W., 1980. Relation between trophic position and Mercury accumulation among fishes from the Tongue River reservoir, Montana. Environmental Research, 22:73-80.

REUTHER, R., 1994. Mercury accumulation in sediments and fish from rivers affected by alluvial gold mining in the Madeira river basin, Brazil. Ambio, 19:11-15.

RODRIGUES, R. M.; MASCARENHAS, A. F. S.; ICHIARA, A. H.; SOUZA, T. M. C.; BIDONE, E. D.; BELLIA, V.; HACON, S.; SILVA, A. R. B.; BRAGA, J. B. \& FILHO, B. S., 1994. Introdução. In: Estudo dos Impactos Ambientais Decorrentes do Extrativismo Mineral e Poluição Mercurial no Tapajós - PréDiagnóstico (E. D. Bidone \& R. M. Rodrigues, org.), pp. 1-28, Rio de Janeiro: Editora Cetem/CNPq.

SANTOS, E. O.,1993. Garimpagem e saúde. In: Conseqüências da Garimpagem no Âmbito Social eAmbiental da Amazônia (A. Mathis \& R. Rehaag, org.), pp. 36-37, Belém: Editora Cejup.

SILVA, A. P., 1993. As diversas formas de garimpo de ouro, suas emissões de mercúrio e mecanismos de dispersão nos diversos compartimentos ambientais. In: Conseqüências da Garimpagem no Âmbito Social eAmbiental da Amazônia (A. Mathis \& R. Rehaag, org.), pp. 48-56, Belém: Editora Cejup.

WESTOO, G., 1966. Determinations of Methylmercury compounds in foodstuffs. Acta Chemica Scandinavica, 22:2131-2137.

WHO (World Health Organization), 1990. Environmental Health Criteria 101: Methylmercury. Geneva: United Nations Environment Program/ International Labour Organization/ World Health Organization. 